$\underline{\text { Preprint typeset in JHEP style. - HYPER VERSION }}$

PCCF-RI-0216

CPPM-P-2002-02

\title{
Neutralino Dark Matter beyond CMSSM Universality
}

\author{
Vincent Bertin ${ }^{1}$, Emmanuel Nezri ${ }^{12}{ }^{2}$, Jean Orloff ${ }^{2}$ \\ ${ }^{1}$ Centre de Physique des Particules de Marseille \\ IN2P3-CNRS, Université de la Méditerranée, F-13288 Marseille Cedex 09 \\ ${ }^{2}$ Laboratoire de Physique Corpusculaire de Clermont-Ferrand \\ IN2P3-CNRS, Université Blaise Pascal, F-63177 Aubiere Cedex
}

\begin{abstract}
We study the effect of departures from SUSY GUT universality on the neutralino relic density, and both its direct and indirect detection, especially by neutrino telescopes. We find that the most interesting models are those with a value of $\left.M_{3}\right|_{G U T}$ lower than the universal case.
\end{abstract}

KEYwords: Beyond Standard Model, SUSY GUT models, Non-universality, Neutralinos, Dark Matter, Detection. 


\section{Contents}

1. Introduction - CMSSM summary 1

2. Non-universal SUSY GUT models 5

3. Non-universality of scalar soft masses and of trilinear couplings at $M_{G U T}$

4. Non-universality of gaugino soft masses at $M_{G U T}$

$4.1 S U(5)$ representations 7

4.2 Free relations in gaugino mass parameters : effect of $\left.M_{3}\right|_{\text {GUT }} \quad 9$

5. Low energy effective parameterisation of the MSSM 17

6. Conclusion 19

\section{Introduction - CMSSM summary}

In a supersymmetric framework with $R$-parity conservation, the lightest supersymmetric particle (LSP) is stable. In the Minimal Supersymmetric Standard Model (MSSM), it is often the lightest neutralino ( $\equiv$ the neutralino $\chi$ ) which is a neutral Majorana particle. It then offers an interesting candidate to account for cold dark matter $(\mathrm{CDM})$ in the present Universe $\left(\Omega_{C D M} \sim 0.3\right)$. The relic population of these neutralinos which survive after spatial separation freezes their self-annihilation, could be detected by the energy they transfer to nuclei in direct detection experiments. Another possibility is the indirect detection of fluxes coming from the decays of neutralino annihilation products $\left(\chi \chi \rightarrow X \bar{X} \rightarrow \nu, \gamma, e^{+} \ldots\right)$ (for a review on neutralino dark matter and different detection possibilities see [四). Since these fluxes go like the squared neutralino density, they require some concentration to restart their annihilation. The gravitational concentration achieved in galactic halos is much too low for neutrino indirect detection. However, neutralinos can also accumulate in the gravitational well of massive astrophysical bodies, and the larger concentration achieved in the centre of the Sun can give rise to higher muon neutrino fluxes detectable by neutrino telescopes such as Antares [2] or IceCube [3].

In a previous paper [1] we studied the potential detection of neutralino dark matter by neutrino telescopes and direct detection experiments in CMSSM models (also 
known as mSugra). Those models inspired from minimal supergravity and gravitymediated SUSY breaking assume a unification of the soft parameters of the MSSM at high energy $M_{G U T} \sim 2 \times 10^{16} \mathrm{GeV}$ reducing the 106 "SUSY" parameters of the MSSM down to 5 : universal masses for scalars $\left(m_{0}\right)$ and gauginos $\left(m_{1 / 2}\right)$, universal trilinear $\left(A_{0}\right)$ and bilinear $\left(B_{0}\right)$ couplings, and a Higgs "mass" parameter $\left(\mu_{0}\right)$. Using renormalisation group equations (RGE) and requiring radiative electroweak supersymmetry breaking, the usual input parameters are $m_{0}, m_{1 / 2}, A_{0}, \tan \beta$ (the ratio of the 2 Higgs doublet vacuum expected values at low energy) and $\operatorname{sign}(\mu)$. The neutralinos are the mass eigenstates coming from the mixing of neutral gauge and Higgs boson superpartners. In the MSSM the neutralino mass matrix in the $\left(\tilde{B}, \tilde{W}^{3}, \tilde{H}_{1}^{0}, \tilde{H}_{2}^{0}\right)$ basis is :

$$
M_{N}=\left(\begin{array}{cccc}
M_{1} & 0 & -m_{Z} \cos \beta \sin \theta_{W} & m_{Z} \sin \beta \sin \theta_{W} \\
0 & M_{2} & m_{Z} \cos \beta \cos \theta_{W} & -m_{Z} \sin \beta \cos \theta_{W} \\
-m_{Z} \cos \beta \sin \theta_{W} & m_{Z} \cos \beta \cos \theta_{W} & 0 & -\mu \\
m_{Z} \sin \beta \sin \theta_{W} & -m_{Z} \sin \beta \cos \theta_{W} & -\mu & 0
\end{array}\right)
$$

and can be diagonalised by a single mixing matrix $z: M_{\text {diag }}=z M_{N} z^{-1}$. The (lightest) neutralino is then given by the linear combination

$$
\chi=z_{11} \tilde{B}+z_{12} \tilde{W}^{3}+z_{13} \tilde{H}_{1}^{0}+z_{14} \tilde{H}_{2}^{0}
$$

In CMSSM models, the neutralino exhibits two different natures: it is fully binolike $\left(z_{11} \approx 1\right)$ for low $m_{0}$ values because of RGE evolution of parameters in eq.(1.1), and can acquire a non-negligible higgsino component for $m_{0} \gtrsim 1000 \mathrm{GeV}$ along the boundary where radiative electroweak symmetry breaking cannot be achieved because of the focus point behaviour [5]. In a previous work [4], we used Suspect $^{1}$ [6, 7] for RGE, potential minimisation and SUSY spectrum calculations, and DarkSusy [8] to estimate relic density and detection rates. We carefully analysed the dominant annihilation channels which count both in relic density calculation and neutrino spectra. In regions where the neutralino relic density satisfy current cosmological constraints, we found that:

- the low $m_{0}$ and $m_{1 / 2}$ region is strongly constrained by experimental limits on SUSY contributions to the $b \rightarrow s+\gamma$ branching ratio, on the lightest Higgs mass and on SUSY contributions to $(g-2)_{\mu}: a_{\mu}^{S U S Y}$,

- $\chi \tilde{t}$ and $\chi \tilde{\tau}$ coannihilation regions are beyond reach of detection,

- the pseudo-scalar $A$ pole region can only be potentially detected with very big future projects for direct detection ( $\sim 1$ ton size),

\footnotetext{
${ }^{1}$ Suspect has been recently updated. This improvement does not affect our results.
} 


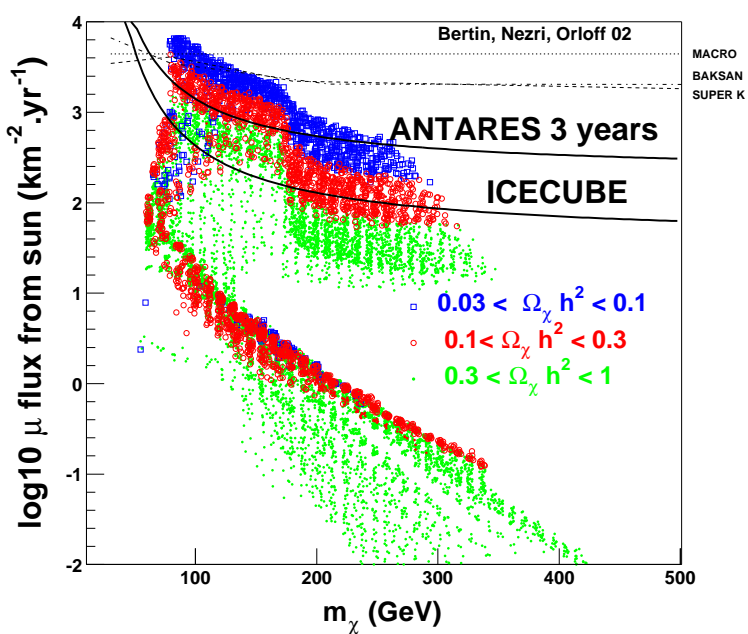

a)

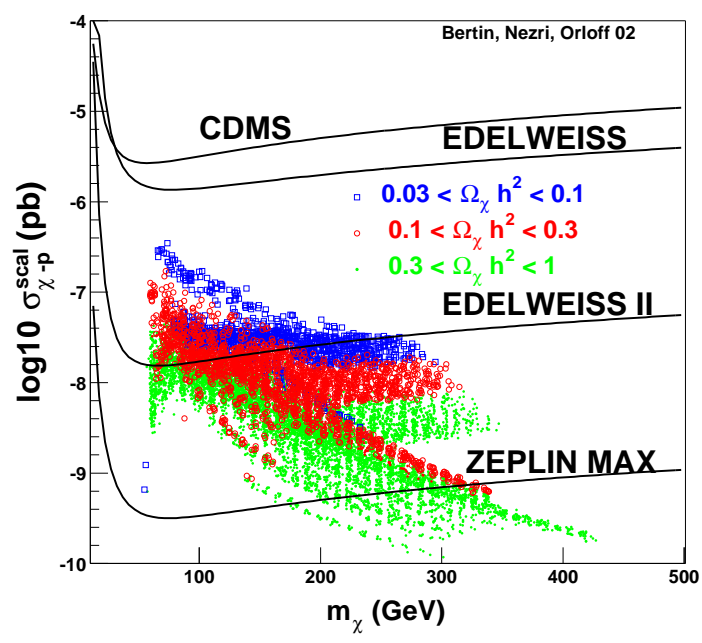

b)

Figure 1: a) Neutrino indirect detection experimental (Macro [9], Baksan [10], SuperKamiokande [11], Antares [12], IceCube [13]) sensitivities on muon fluxes with a $5 \mathrm{GeV}$ threshold coming from $\chi$ annihilations in the Sun and b) direct detection experimental

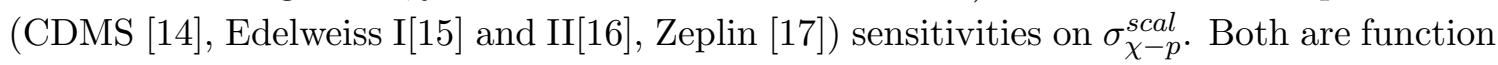
of the neutralino mass for a wide scan of CMSSM models (see text for details).

- the large $m_{0}$ "focus point" region where the neutralino has a significant (and crucial, see figure 2 $\mathrm{b}$ ) higgsino fraction $\left(f_{H}\right)$ is interesting for direct and indirect detection experiments. Neutrino/muon fluxes coming from the Sun are large only in this region because of the $\chi \chi \stackrel{\chi_{i}^{+}, \chi_{i}}{\rightarrow} W^{+} W^{-}, Z Z$ and $\chi \chi \stackrel{Z}{\rightarrow} t \bar{t}$ channels which give rise to more energetic neutrino spectra and to muons with higher energy.

This is summarised on figures 1 and 2 for the following sample of CMSSM models, compared to sensitivities of current and future direct and neutrino indirect detection experiments :

- $0<m_{0}<3000 \mathrm{GeV} ; 40<m_{1 / 2}<800 \mathrm{GeV} ; A_{0}=0 \mathrm{GeV} ; \tan \beta=10,50$; $\mu>0$

- $0<m_{0}<3000 \mathrm{GeV} ; 40<m_{1 / 2}<1000 \mathrm{GeV} ; A_{0}=-800,-400,0,400,800$ $\mathrm{GeV} ; \tan \beta=20,35 ; \mu>0$,

- $0<m_{0}<3000 \mathrm{GeV} ; 40<m_{1 / 2}<1000 \mathrm{GeV} ; A_{0}=0 \mathrm{GeV} ; \tan \beta=45$; $\mu>0$.

The constraints we apply on the models are : 


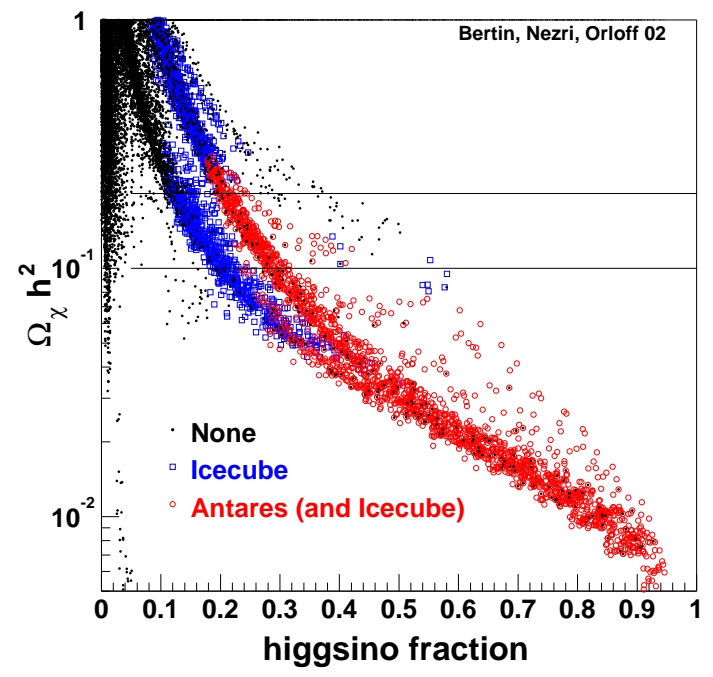

a)

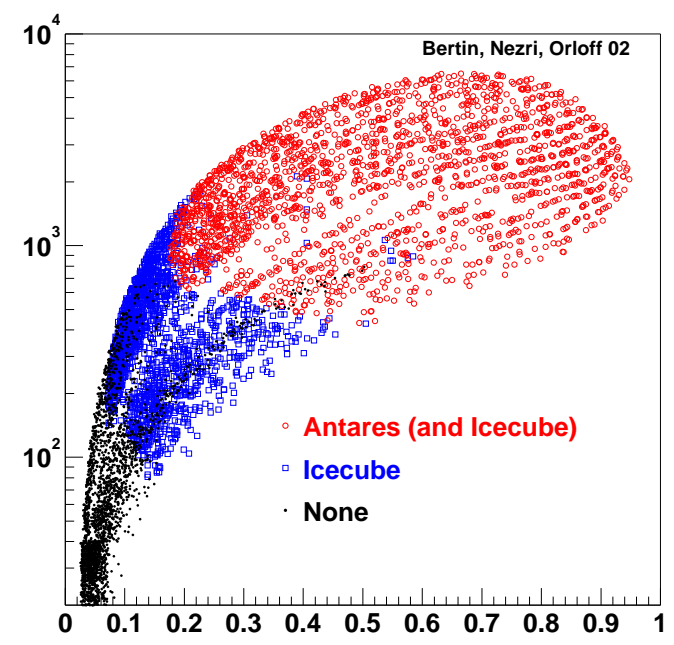

b)

Figure 2: a) Neutralino relic density and b) muon flux coming from the Sun as functions of the $\chi$ higgsino fraction for a wide scan of CMSSM models (see text for details). The band in plot a) indicates the current preferred range for $\Omega_{C D M}$.

- limits on SUSY particle masses from accelerators searches (see e.g. 18 to update these values):

$m_{\chi_{1}^{+}}>104 \mathrm{GeV} ; m_{\tilde{f}}>100 \mathrm{GeV}$ for $\tilde{f}=\tilde{t}_{1}, \tilde{b}_{1}, \tilde{l}^{ \pm}, \tilde{\nu}, m_{\tilde{g}}>300 \mathrm{GeV} ; m_{\tilde{q}_{1,2}}>$ $260 \mathrm{GeV}$ for $\tilde{q}=\tilde{u}, \tilde{d}, \tilde{s}, \tilde{c}$,

- limits on Higgs mass [19] : we require $m_{h}>112 \mathrm{GeV}$ instead of $m_{h}>114$ $\mathrm{GeV}$ (depending on $\tan (\beta-\alpha)$ ) because of the too low value for Higgs mass in Suspect $2.005(\sim 3 \mathrm{GeV})$ as pointed out in [20],

- limits on $b \rightarrow s \gamma$ branching ratio : we require $B R(b \rightarrow s+\gamma)=1 \rightarrow 4 \times 10^{-4}$ as calculated in DarkSusy, corresponding to $B R(b \rightarrow s+\gamma) \simeq 2.3 \rightarrow 5.3 \times 10^{-4}$ (the Standard Model value calculated in DarkSusy is $2.4 \times 10^{-4}$ instead of the NLO $3.6 \times 10^{-4}$ [21]) when experimental results are [22] $B R(b \rightarrow s+\gamma)=$ $3.37 \pm 0.37 \pm 0.34 \pm 0.24 \pm 0.38 \times 10^{-4}$.

- limits on SUSY contribution to muon anomalous moment $(g-2)_{\mu}[23]:-6 \times$ $10^{-10}<a_{\mu}^{S U S Y}<58 \times 10^{-10}$.

The Renormalisation Group Equations (RGE) lead SUSY models to a generic hierarchy of particle spectrum in which scalars are heavier than light neutralinos and charginos. As summarised above, the most interesting CMSS Models for the detection of a relic neutralino are those for which its higgsino fraction is non negligible and has a dominant effect (figure 2). These are indeed the only models leading to 
large neutralino annihilation cross section $\sigma_{\chi-\chi}^{A}$ (important for its relic density and for indirect detection) and to large neutralino-proton scalar and spin dependent elastic cross sections (important for direct and indirect detection). In this paper, using the same tools as previously [6, 8], we shall relax some universality hypotheses and examine whether more favourable models for relic density and detections (both direct and neutrino telescopes) can be found, and establish that models detectable by neutrino telescopes are more generic than the "focus point" region of a typical $\left(m_{0}, m_{1 / 2}\right)$ CMSSM plane, offering a less constrained framework for detection. We will then conclude by a generic low energy parameterisation of typical models coming from RGE evolutions, favourable for neutrino indirect detection.

\section{Non-universal SUSY GUT models}

In which direction can one relax the universality hypothesis in order to induce the physics we want, namely to get large $\sigma_{\chi-\chi}^{A}, \sigma_{\chi-q}^{\text {scal }}$ and $\sigma_{\chi-q}^{\text {spin }}$ and thus a non negligible higgsino fraction for the neutralino ?

We can start with some arguments on non-universality inspired by the (1-loop) renormalisation group equations [24]. It has been shown 25] that from the RGE solutions of the couplings in the unbroken symmetry phase, one can obtain the soft terms of the broken phase by an expansion over Grassman variables. This has been done for the MSSM couplings [24] in order to get the RGE solutions for the soft terms. In the case of an analysis with three independent Yukawa couplings $h_{t}, h_{b}$ and $h_{\tau}$, it is found that due to the infra-red quasi fixed point (IRQFP) [26] behaviour of the Yukawa couplings, the low energy values of the third generation scalar soft masses and of the Higgs masses have a weak (scale independent) relation with their initial values at $M_{G U T}$ and depend mainly on the high energy $S U(3)$ gaugino soft mass $\left.M_{3}\right|_{G U T}$ (in a scale dependent way). The first and second generation soft masses have an analogous behaviour because of their negligible Yukawa couplings. This can be written as :

$$
\left(\left.M_{\text {soft }}^{\text {scal }}\right|_{\text {low }}\right)^{2}=\left(\left.M_{\text {soft }}^{\text {scal }}\right|_{G U T}\right)^{2}+c_{3} f_{3}+c_{2} f_{2}+c_{1} f_{1}+\text { corrections }
$$

with

$$
f_{i}=\frac{\left(M_{i}^{G U T}\right)^{2}}{b_{i}}\left(1-\frac{1}{\left(1+b_{i} \alpha_{0} t\right)^{2}}\right),
$$

where $M_{i}$ are the soft gaugino mass terms and $\alpha_{0}$ is the universal gaugino coupling at $M_{G U T}$, and where $c_{3}$ is strongly dominant for squarks and Higgses and less dominant for sleptons which have vanishing $S U(3)$ charges.

In addition, a gaugino non-universality given by $\left(M_{2} / M_{1}\right)_{G U T}<1$ can lead to a large wino component for the neutralino and to an important modification of its couplings with respect to the universal CMSSM case and thus to a very different phenomenology. 
We will now explore various non-universal scenarios in order to study their possible benefits on detection.

\section{Non-universality of scalar soft masses and of trilinear cou- plings at $M_{G U T}$}

\section{Sfermions :}

As for $A_{0}$ in the CMSSM [4], modifications of the soft parameters in the sfermions mass matrices can give rise to light third generation sfermions. This could modify the neutralino relic density value through coannihilation processes. However, in detection processes, the neutralino interacts with nuclei and thus mainly with $u$ and $d$ valence quarks. Due to their small Yukawa couplings, RGE evolutions of the first and second generation squark masses only depend on gaugino soft masses. This implies that their masses can not be lowered by changing scalar soft terms to enhance $\sigma_{\chi-q}^{\text {scal }}$ and $\sigma_{\chi-q}^{\text {spin }}$

through the process $\chi q \stackrel{\tilde{q}}{\rightarrow} \chi q$. Non-universality in the sfermion mass soft terms can thus only lead to $\chi \tilde{\tau}$ [27, $\chi \tilde{t}$ [28, 29, 30] coannihilation effects, giving rise to models for which the neutralino is cosmologically a good dark matter candidate without modifying its detectability.

The soft terms for the third generation sfermions being present in soft Higgs masses RGE's $\left(X_{t}, X_{b}\right.$ and $\left.X_{\tau}\right)$ can also influence the radiative electroweak symmetry breaking through the running of $m_{H_{1}}^{2}$ and $m_{H_{2}}^{2}$. This effect is similar to the one discussed in the next section. One could also add lighter third generation squarks but this is not helpful for detection.

\section{Higgses :}

One can relax the universality relation for the Higgs soft masses at the Grand Unification scale $\left.m_{H_{1}}\right|_{G U T}=\left.m_{H_{2}}\right|_{G U T}=m_{0} \cdot m_{H_{1}}$ and $m_{H_{2}}$ being now free parameters, the potential minimisation and thus the value of $\mu$ is less constrained. The parameterisation usually taken is [31, 32, 33, 34 :

$$
\left.m_{H_{i}}\right|_{G U T}=\left(1+\delta_{i}\right) m_{0} ; \text { for } i=1,2 \text {. }
$$

Due to the departure of the Higgs soft masses with respect to the universal case, $\mu$ values are modified and the parameter space of a typical $\left(m_{0}, m_{1 / 2}\right)$ plane satisfying the radiative electroweak symmetry breaking is reduced with respect to the CMSSM. In the remaining parameter space, models with smaller masses for the heavier Higgs bosons $m_{A}$ and $m_{H}$ are more easily obtained than for the (universal) CMSSM, leading to wider zones with a good neutralino relic density and accessible direct detection yields. Concerning the neutrino indirect detection potential, the muon fluxes due to neutralino annihilation in the Sun remain however small for such models. This is in contradiction with the recent results presented in ref.[33], in which the modification of the Higgs soft mass relations generate more easily models with large muon 


$$
m_{H_{2}}=m_{0} ; m_{H_{1}}=0.5 m_{0} ; A_{0}=0 ; \tan \beta=45 ; \mu>0
$$

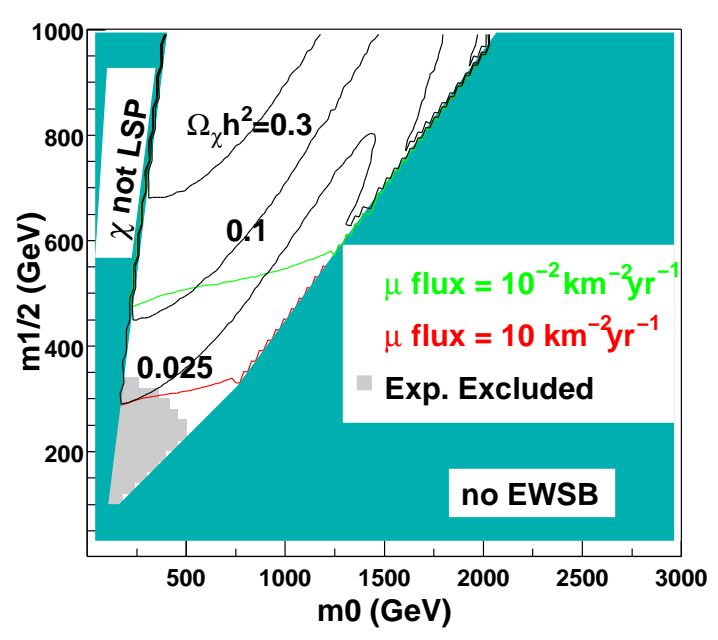

a)

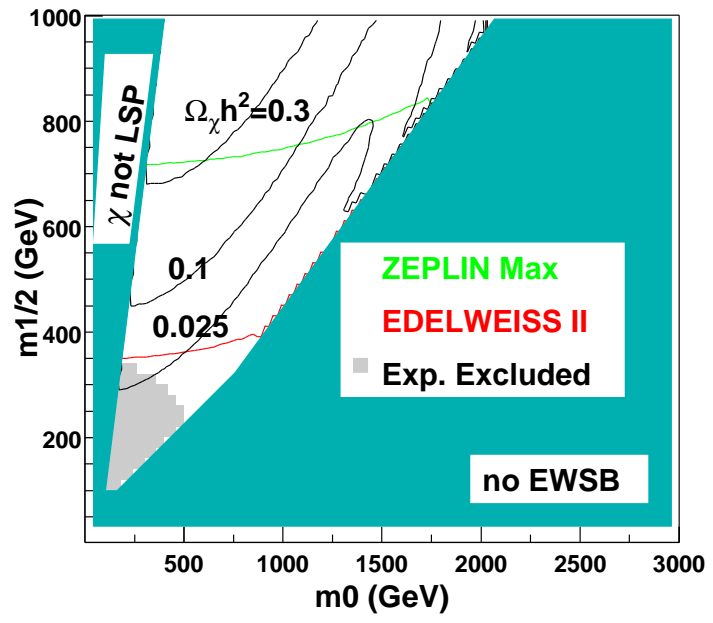

b)

Figure 3: Neutralino detection potential a) on muon fluxes coming from the Sun for neutrino telescopes and $\mathrm{b}$ ) for direct detection experiments in the $\left(m_{0}, m_{1 / 2}\right)$ plane for models with non-universal Higgs soft masses $m_{H_{2}}^{2}=m_{0}$ and $m_{H_{1}}^{2}=0.5 m_{0}$ and with $A_{0}=0$, $\tan \beta=45, \mu>0$. Also shown are constant relic density lines $\Omega_{\chi} h^{2}=1,0.3,0.1,0.025$. The small grey area shows the models excluded by current experimental constraints.

fluxes coming from the Sun in the parameter space allowed by radiative electroweak symmetry breaking. This difference can certainly be attributed to the use in [33] of IsaSusy (version < 7.64) for RGE's and SUSY spectrum calculation (instead of Suspect in this work) for which the predicted value of $\mu$ is usually too small for high $m_{0}$ values [20, and hence the higgsino fraction of the neutralino is too large. An example of our results is shown on figure 3 for $\delta_{1}=-0.5$ and $\delta_{2}=0$.

\section{Non-universality of gaugino soft masses at $M_{G U T}$}

\section{1 $S U(5)$ representations}

In CMSS Models, a universal mass is given to all gaugino fields at the high energy scale $M_{G U T}$. The unification gauge group should either be $S U(5)$ or a larger group naturally breaking into an $S U(5)$ subgroup at a scale around $M_{G U T}$. In the class of models for which the SUSY breaking is induced by an $F$-term, the gaugino masses are generated by a chiral superfield with an auxiliary component $F_{\Phi}$ acquiring a vev such that [35, 36, 37]

$$
\mathcal{M}_{\text {gauginos }} \sim \frac{<F_{\Phi}>_{a b}}{M_{\text {Planck }}} \lambda^{a} \lambda^{b}
$$




\begin{tabular}{|c|ccc|ccc|}
\hline & \multicolumn{3}{|c|}{$M_{G U T}$} & \multicolumn{3}{c|}{$m_{Z}$} \\
$F_{\Phi}$ & $M_{3}$ & $M_{2}$ & $M_{1}$ & $M_{3}$ & $M_{2}$ & $M_{1}$ \\
\hline $\mathbf{1}$ & 1 & 1 & 1 & $\sim 6$ & $\sim 2$ & $\sim 1$ \\
$\mathbf{2 4}$ & 2 & -3 & -1 & $\sim 12$ & $\sim-6$ & $\sim-1$ \\
$\mathbf{7 5}$ & 1 & 3 & -5 & $\sim 6$ & $\sim 6$ & $\sim-5$ \\
$\mathbf{2 0 0}$ & 1 & 2 & 10 & $\sim 6$ & $\sim 4$ & $\sim 10$ \\
\hline
\end{tabular}

Table 1: Relative values of the gaugino masses at $M_{G U T}$ and $m_{Z}$ scales in the four possible irreducible representations for $F_{\Phi}$.

where $\lambda^{a}$ and $\lambda^{b}$ are the gaugino fields $\tilde{B}, \tilde{W}$ and $\tilde{g}$. Since all gaugino fields belong to the adjoint representation, $F_{\Phi}$ belongs to an irreducible representation in the symmetrised product of two adjoints of $S U(5)(=\mathbf{2 4})$ or to a linear combination of these representations :

$$
(24 \times 24)_{\text {sym }}=1 \oplus 24 \oplus 75 \oplus 200
$$

where only the singlet component (1) leads to universal masses $\left.M_{1}\right|_{G U T}=\left.M_{2}\right|_{G U T}=$ $\left.M_{3}\right|_{G U T}=m_{1 / 2}$. The embedding coefficients of the Standard Model gauge groups in $S U(5)$ give the relations between the gaugino masses at $M_{G U T}$ (see table 1), which we now discuss for the pure non-singlet irreducible representations.

\section{Case of the 24 :}

For low $m_{0}$ values, since $M_{1}^{24} / M_{2}^{24}<M_{1}^{C M S S M} / M_{2}^{C M S S M} \sim 1 / 2$ at low energy, the neutralino is strongly bino-like. As for the CMSSM, this kind of models will not lead to large indirect detection muon fluxes coming from the Sun. In addition, the lower limits on the neutralino mass obtained by LEP experiments are only valid for $M_{1} / M_{2} \geq 1 / 2$ at low energy, so models with a very light neutralino are not excluded. But the main grey bubble part of the exclusion domain comes from the lower limits on the SUSY contribution to the muon $g-2$ parameter $a_{\mu}^{S U S Y}$ which are very strong for these models.

For large $m_{0}$ values, a small higgsino fraction in the neutralino can be obtained in the focus point region, but less easily than in the CMSSM universal case because the larger $\left|M_{2}\right|$ value leads to larger $m_{H_{2}}^{2}$ values. So the direct detection yields and indirect detection muon fluxes for these models are less important than for the universal case and the neutralino relic density is too large. This is illustrated in figure 4 , where no model is accessible to the Antares sensitivity, and where the very thin IceCube detection region always leads to a cosmic over-weight. In addition, the Edelweiss II and Zeplin detection regions are strongly reduced with respect to the universal case for large $m_{0}$ values, due to the small higgsino fraction of the neutralino, and also due to the relative sign between $M_{1}, M_{2}$ and $\mu$ (same effect as $\mu<0$ for the CMSSM with the "hole" in the spin independent elastic cross section due to the suppression between up and down quarks contributions [38]). 
$24: A_{0}=0 ; \tan \beta=45 ; \mu>0$

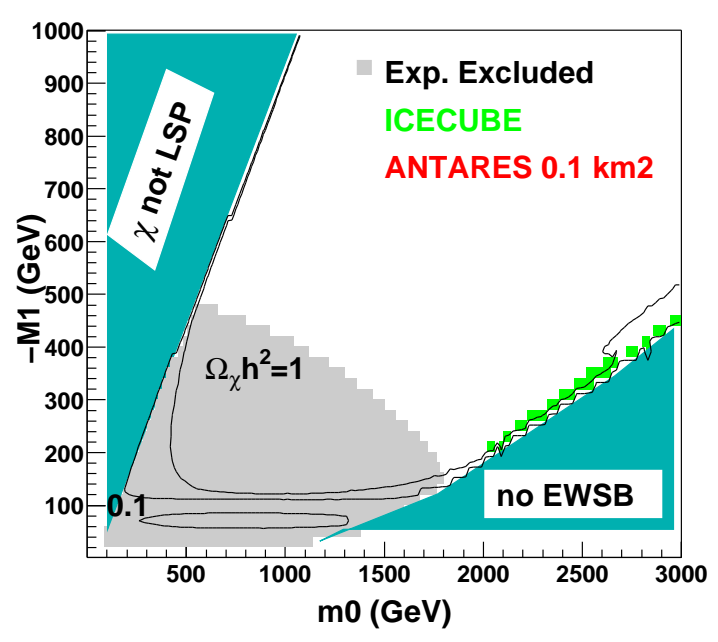

a)

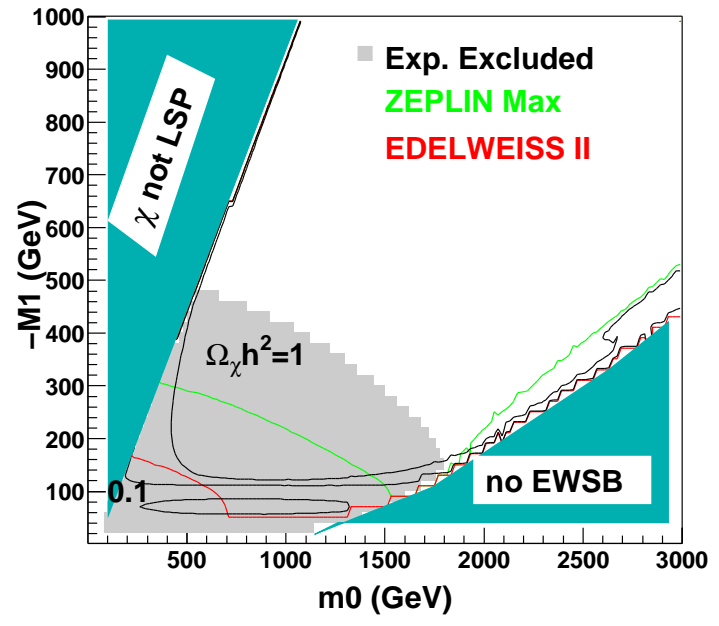

b)

Figure 4: Neutralino detection potential a) for neutrino telescopes detecting muon fluxes coming from the Sun and b) for direct detection experiments in the $\left(m_{0},-M_{1}\right)$ plane of the 24 representation for $A_{0}=0, \tan \beta=45, \mu>0$. No model with a good relic density is accessible to Antares $\left(0.1 \mathrm{~km}^{2}\right)$.

This is in agreement with the results of ref. 39] in which the neutralino has been chosen even more bino-like, $M_{2} / M_{1} \sim 10$, leading to a relic over-density except for light sleptons.

Case of the 75 :

At low energy $\left|M_{1}\right| \sim\left|M_{2}\right| \sim\left|M_{3}\right|$, the neutralino is thus equally wino and bino, and the two lightest neutralinos and the lightest chargino are almost degenerate $M_{\chi} \sim M_{\chi_{2}^{0}} \sim M_{\chi_{1}^{+}}$. These models open up the very efficient $\chi \chi \stackrel{\chi_{1}^{+}, \chi_{2}^{0}}{\longrightarrow} W^{+} W^{-}, Z Z$ annihilation channels, as well as very strong $\chi \chi_{2}^{0}$ and $\chi \chi_{1}^{+}$coannihilations. This leads to a strong reduction of the relic density. In addition, the gluino can sometimes be the LSP.

Case of the 200 :

Here $\left|M_{2}\right| \sim 2 / 5\left|M_{1}\right|$ at low energy giving a strongly wino-like neutralino. $\chi \chi \stackrel{\chi_{1}^{+}, \chi_{2}^{0}}{\longrightarrow}$ $W^{+} W^{-}, Z Z$ annihilations and $\chi \chi_{1}^{+}$coannihilation totally suppress the neutralino population.

\subsection{Free relations in gaugino mass parameters : effect of $\left.M_{3}\right|_{G U T}$}

As explained above, the MSSM non-universality parameters giving the strongest impact on the detection of dark matter neutralinos are $\left.M_{2}\right|_{G U T}$ and mostly $\left.M_{3}\right|_{G U T}$. We will now study the departure from universality of these two parameters and their 
benefits on the neutralino relic density and the detection yields. These non-universal values will then be translated in the above $S U(5)$ representation decomposition.

$m_{0}=1500 ; m_{1 / 2}=600 ; A_{0}=0 ; \tan \beta=45 ; \mu>0$

a)
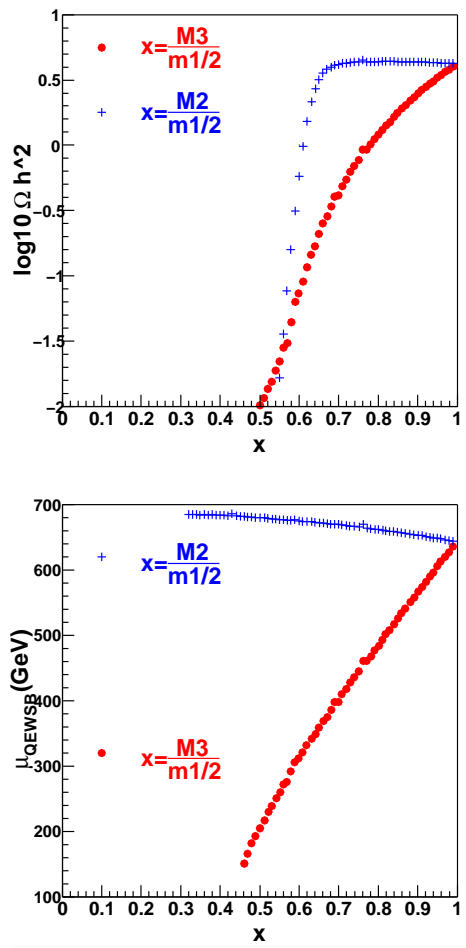

d)
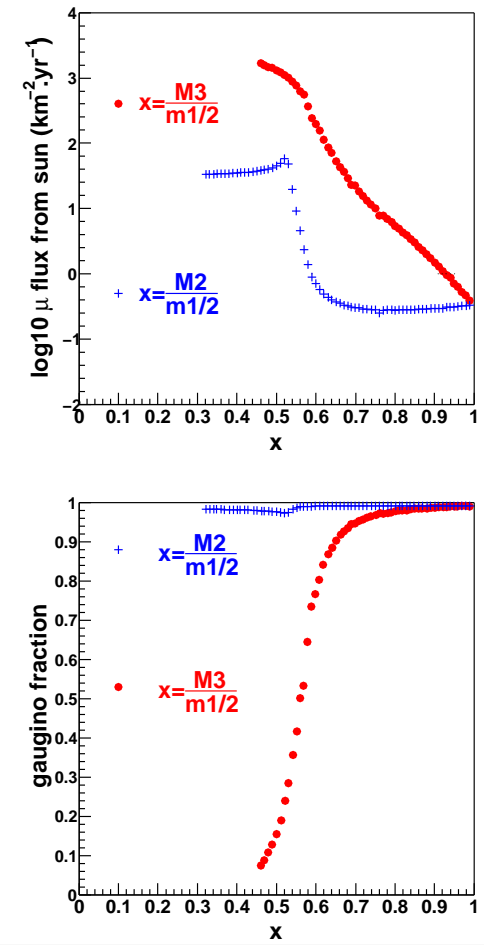

e)
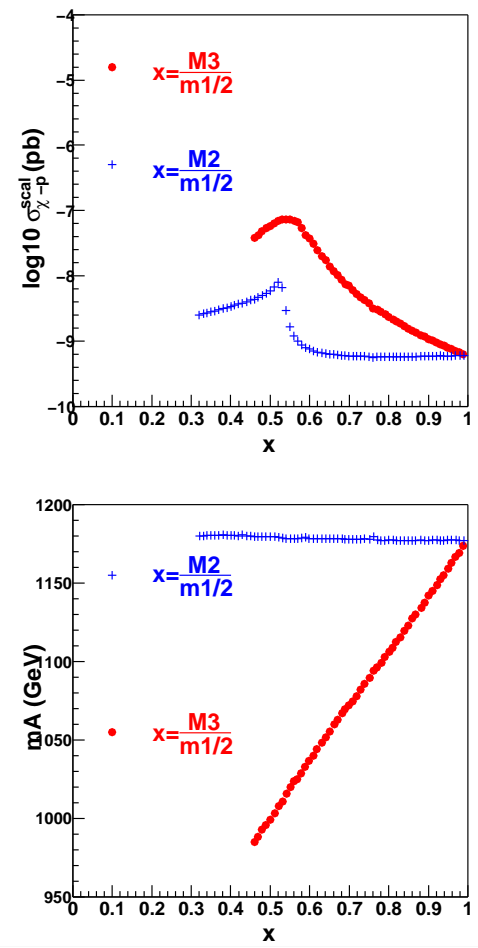

f)

Figure 5: Evolution of a) the neutralino relic density, b) the muon flux coming from the Sun, c) the spin independent neutralino-proton cross section (direct detection), d) the $\mu$ parameter, e) the gaugino fraction and $\mathrm{f}$ ) the pseudo-scalar mass $m_{A}(\mathrm{f})$ as functions of the $\frac{M_{2}}{m_{1 / 2}}$ and $\frac{M_{3}}{m_{1 / 2}}$ ratios for the CMSS Model with $m_{0}=1500 \mathrm{GeV}, m_{1 / 2}=600 \mathrm{GeV}$, $A_{0}=0 \mathrm{GeV}, \tan \beta=45, \mu>0$.

In the following, the departure from universality effects are quantified by the ratios at $M_{G U T} x=M_{2} / m_{1 / 2}$ or $x=M_{3} / m_{1 / 2}\left(\right.$ with $m_{1 / 2}=M_{1}=M_{3}$ or $m_{1 / 2}=$ $M_{1}=M_{2}$ respectively) which will be lowered starting from the CMSSM case $(x=1)$. The $\left.M_{2}\right|_{G U T}$ parameter:

The effect of the $M_{2}$ parameter is essentially a modification of the neutralino composition. When the wino component of the neutralino increases, the $\chi \chi \stackrel{\chi_{1}^{+}, \chi_{2}^{0}}{\longrightarrow}$ $W^{+} W^{-}, Z Z$ processes become more effective and enhance the annihilation cross section $\sigma_{\chi-\chi}^{A}$ [40]. In addition, the strong $\chi \chi_{2}^{0}$ and $\chi \chi_{1}^{+}$coannihilations become active and the neutralino relic density strongly decreases. This wino component can 
$m_{0}=1500 ; m_{1 / 2}=600 ; A_{0}=0 ; \tan \beta=10 ; \mu>0$

a)
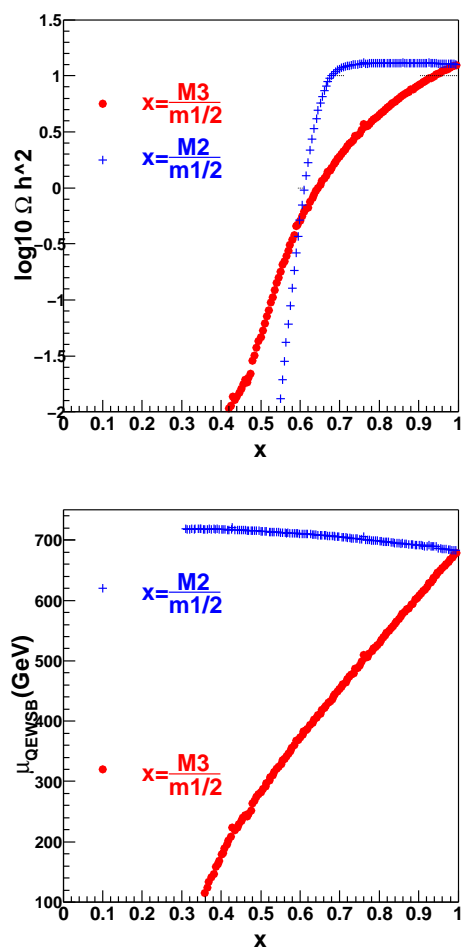

d) b)
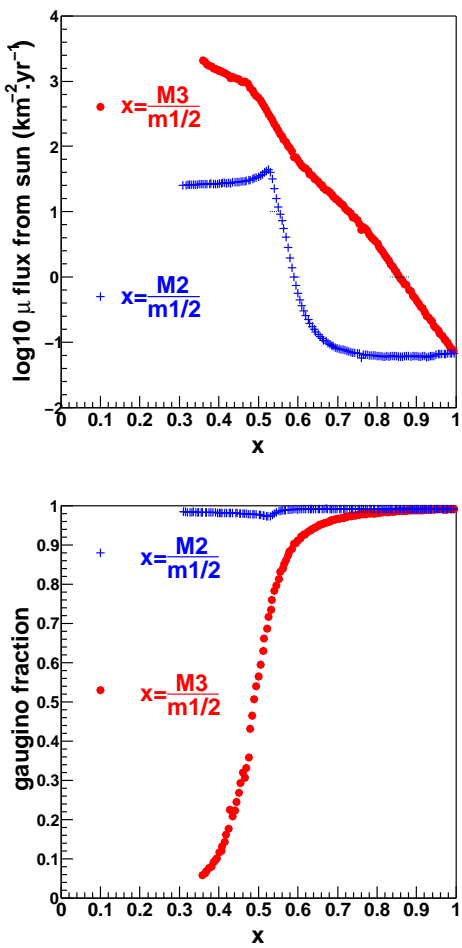

e) c)
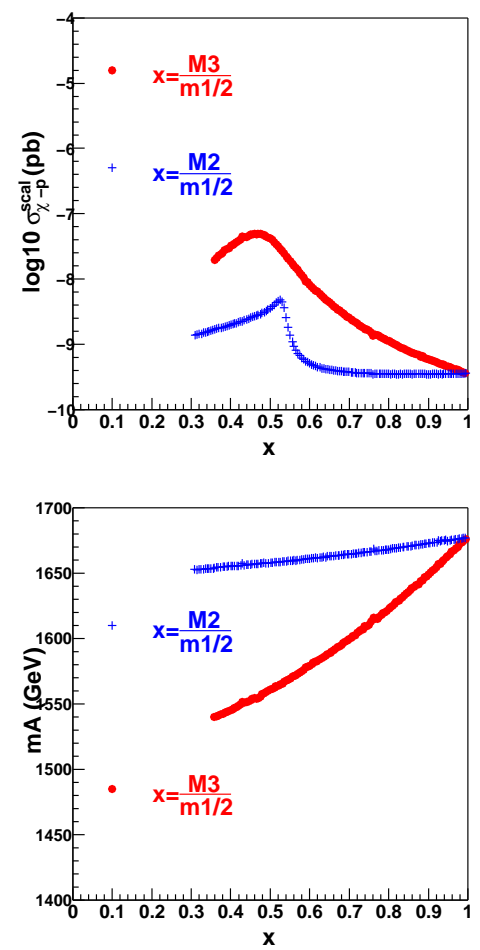

f)

Figure 6: Same as figure 5 for $\tan \beta=10$ instead of 45 .

increase detection rates by an order of magnitude at most. Indeed, the neutralinoquark coupling, which enters in direct detection $\left(\sigma_{\chi-q}^{\text {scal }}, H\right.$ exchange $)$ and in the capture for indirect detection $\left(\sigma_{\chi-q}^{\text {spin }}, \tilde{q}\right.$ exchange), is $\tan \theta_{W}$-suppressed for pure bino w.r.t. pure wino. The neutralino annihilations into the hard $W^{+} W^{-}$spectrum also give rise to more energetic muons. These enhancements of the (in)direct detection yields can reach several order of magnitude with respect to the CMSSM case depending on the values of the other SUSY parameters as can be seen in figures 5b and 5c, 6b and 6c, and 7b and 7c.

However, the relevant value of $M_{2}$ is very critical, so its benefits are only operative in a very narrow range. Given an $M_{2} / m_{1 / 2} \sim 0.6-0.7$ ratio (equivalent to $\left.\left.\left.M_{1}\right|_{\text {low }} \sim M_{2}\right|_{\text {low }}\right)$, the neutralino detection yields are enhanced but the relic density drops down to much too small values (see figures 5a, 6a and 7a ). In conclusion, the handling of this $M_{2}$ parameter in order to get the desired neutralino dark matter phenomenology can only be done by "fine-tuning". One way around this wino neutralino extermination suggested in [41] is to have the wino neutralino population derived from AMSB models $\left(\left.\left.M_{1}\right|_{\text {low }} \simeq 3 M_{2}\right|_{\text {low }}\right)$ regenerated at low temperature by 
moduli decays which could give a good relic abundance.

\section{The $\left.M_{3}\right|_{G U T}$ parameter:}

The impact of variations in the $M_{3}$ parameter is much more interesting. It is indeed one of the key parameters of the MSSM through the RGE's. Its influence goes well beyond the neutralino sector. Indeed, following the RGE [24] (see equation 2.1), a decrease of $\left.M_{3}\right|_{G U T}$ leads to a decrease of $m_{H_{u}}^{2}$ and of $\mu$ through the radiative electroweak symmetry breaking mechanism, thus enhancing the neutralino higgsino fraction, and leads also to a decrease of $m_{\tilde{q}}$ and $m_{A}$. These effects are illustrated on the figures $5 \mathrm{~d}, 5 \mathrm{e}, 5 \mathrm{f}, 6 \mathrm{~d}, 6 \mathrm{e}, 6 \mathrm{f}$ and $7 \mathrm{~d}, 7 \mathrm{de}, 7 \mathrm{f}$. The neutralino relic density then gradually decreases with $x=M_{3} / m_{1 / 2}$ (see figures $5 \mathrm{a}$, 6a and $7 \mathrm{a}$ ) due to the increase of the CMSSM dominant annihilation cross section channel (mainly $\chi \chi \stackrel{A}{\rightarrow} b \bar{b}, \chi \chi \stackrel{Z}{\rightarrow} t \bar{t}$, $\chi \chi \stackrel{\chi_{i}^{+}}{\longrightarrow} W^{+} W^{-}$and $\chi \chi \stackrel{\chi_{i}}{\rightarrow} Z Z$ according to the CMSSM starting parameters $[4]$ ). The annihilation channels which directly depend on the neutralino higgsino fraction finally dominate when $x$ is further lowered, because of the decreasing of $\mu$.

For the CMSS Model with $m_{0}=1500 \mathrm{GeV}, m_{1 / 2}=600 \mathrm{GeV}, A_{0}=0 \mathrm{GeV}$, $\tan \beta=45, \mu>0$ (figures 5 and $8 \mathrm{a}$ ), the dominant channel at $x=1$ is $\chi \chi \stackrel{A}{\rightarrow} b \bar{b}$. By decreasing $x$, the latter remains at first dominant while the neutralino relic density is reduced due to the decrease of $m_{A}$ (figure $5 \mathrm{a}$ and $\mathrm{f}$ ), then since $\mu$ also decreases, the

processes $\chi \chi \stackrel{Z}{\rightarrow} t \bar{t}$ followed by $\chi \chi \stackrel{\chi_{i}^{+}}{\rightarrow} W^{+} W^{-}$and $\chi \chi \stackrel{\chi_{i}}{\rightarrow} Z Z$ take successively over (as well as the $\chi \chi^{+}$and $\chi \chi_{2}^{0}$ coannihilations) further lowering the relic density. The enhancement of the $t \bar{t}$ branching ratio before the increase of the neutralino higgsino fraction $(x>0.8)$ (see figure $8 \mathrm{a})$ is due to the decrease of the stop mass.

For the same CMSS Model but with $\tan \beta=10$ (figures 6 and $8 \mathrm{~b}$ ), $m_{A}$ is larger due to the smaller $\tan \beta$ value, so the $\chi \chi \stackrel{Z}{\rightarrow} t \bar{t}$ channel dominates the neutralino annihilation. When $x$ is lowered, this process remains dominant (figure $8 \mathrm{~b}$ ) but its cross section firstly increases because $m_{\tilde{t}}$ decreases. Then the $Z$ exchange process proportional to the neutralino higgsino fraction takes over due to the decrease of $\mu$ (see figure 6]d ) enhancing the annihilation cross section and decreasing the relic density (see figure 6a ). Finally, the annihilation processes into gauge bosons become dominant for $x \lesssim 0.5$ because $m_{\chi}$ becomes smaller than the top mass.

For the CMSS Model with $m_{0}=3000 \mathrm{GeV}, m_{1 / 2}=2000 \mathrm{GeV}, A_{0}=0 \mathrm{GeV}$, $\tan \beta=45, \mu>0$ (figures 7 and $8 \mathrm{c}$ ), the neutralino relic density is much too large in the CMSSM, but lowering $M_{3}$ drives this model into the cosmologically favoured region by the decrease of $m_{A}$ and $\mu$ (figures $7 \mathrm{a}$, $7 \mathrm{~d}$ and $7 \mathrm{f}$ ). This model is located further away from the radiative electroweak symmetry breaking boundary and has larger $\mu$. In addition, $m_{A}<m_{\tilde{q}}$ so the process $\chi \chi \stackrel{A}{\rightarrow} b \bar{b}$ strongly dominates over $\chi \chi \stackrel{Z, \tilde{t}}{\rightarrow} t \bar{t}$. Again, annihilations into gauge bosons take over when $x$ (i.e. $\mu$ ) decreases. Neutralino direct and indirect detections:

When $x$ is lowered, the direct detection yields (see figures 5c, 6c and $7 \mathrm{c}$ ) can be 
$m_{0}=3000 ; m_{1 / 2}=2000 ; A_{0}=0 ; \tan \beta=45 ; \mu>0$

a)
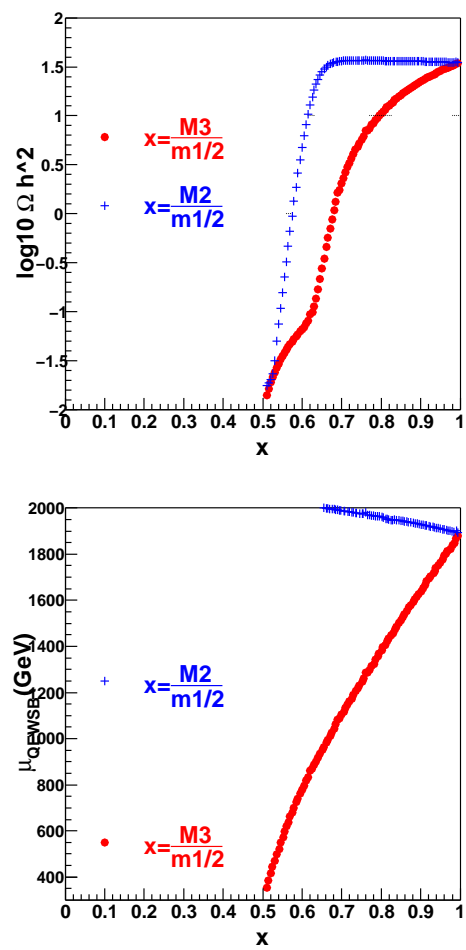

d)
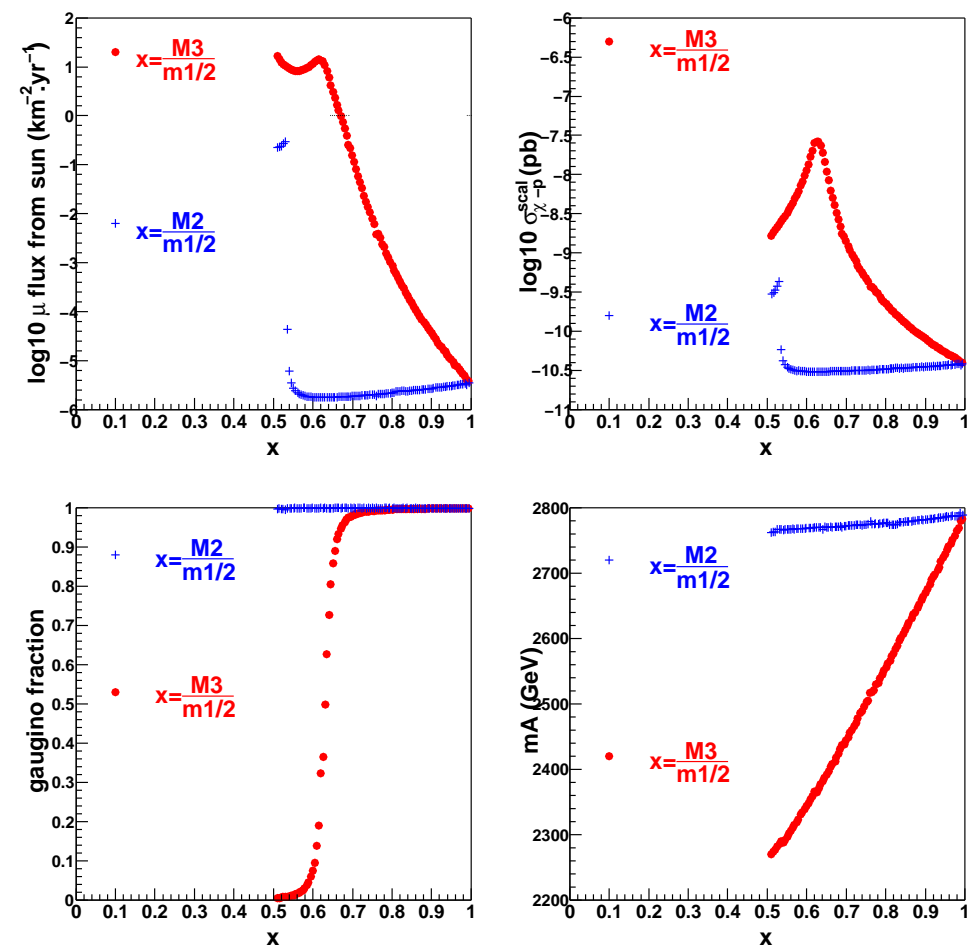

e)

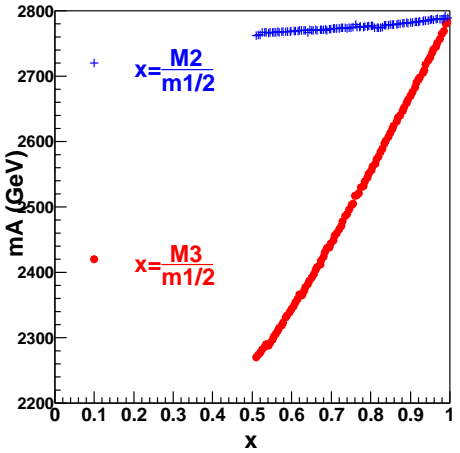

f)

Figure 7: Same as figure 5 for the extreme CMSS Model with $m_{0}=3000 \mathrm{GeV}, m_{1 / 2}=$ $2000 \mathrm{GeV}, A_{0}=0 \mathrm{GeV}, \tan \beta=45, \mu>0$.

$m 0=1500 ; m 1 / 2=600 ; A 0=0 ; \tan (\beta)=45 ; \mu>0$

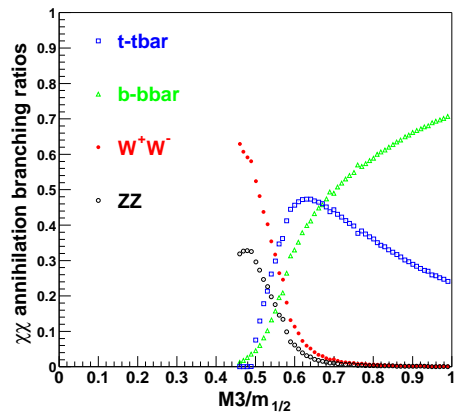

a) $m 0=1500 ; m 1 / 2=600 ; A 0=0 ; \tan (\beta)=10 ; \mu>0$

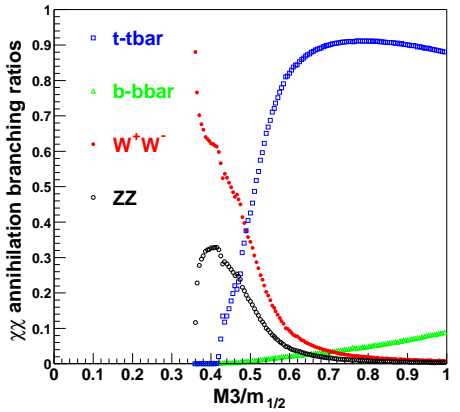

b) $m 0=3000 ; m 1 / 2=2000 ; A 0=0 ; \tan (\beta)=45 ; \mu>0$

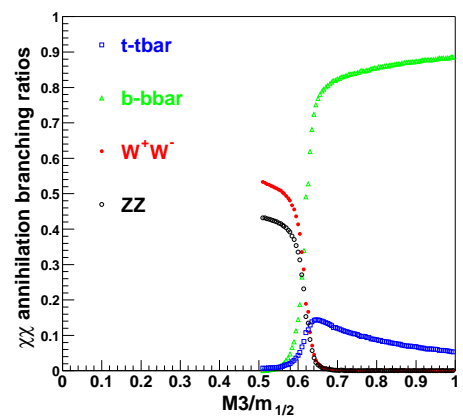

c)

Figure 8: Evolution of the dominant neutralino annihilation branching ratios as functions of the $M_{3} / m_{1 / 2}$ ratio for the models a) of figures 5 , b) of figures 6 and c) of figures 6 . 
enhanced by 2 or 3 orders of magnitude with respect to the CMSSM case $(x=1)$. Firstly the reduction of the squark masses favours the $\chi q \stackrel{\tilde{q}}{\rightarrow} \chi q$ process, and mainly the coupling $C_{\chi q H}$ is maximal for maximum $z_{13(4)} z_{11(2)}$ mixed products which increase with the neutralino higgsino fraction when $x$ decreases. Moreover, $m_{H} \sim m_{A}$ and thus $\sigma_{\chi-p}^{\text {scal }}$ is enhanced due to the decrease of $m_{A}$. When the neutralino gaugino fraction finally drops, the $z_{13(4)} z_{11(2)}$ products decrease and $\sigma_{\chi-p}^{\text {scal }}$ decreases back with $x$. This behaviour can clearly be remarked on the figures 5c, 5e, 5f, 6 c , 6e, 6f and

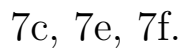

As far as neutrino indirect detection is concerned, the enhancement on the muon fluxes coming from neutralino annihilation in the Sun, due to the decrease of $\left.M_{3}\right|_{G U T}$, can reach up to 6 orders of magnitude with respect to the CMSSM case $(x=1)$. The main effect is coming from the increase of the spin dependent neutralino-proton elastic cross section, firstly due to the decrease of the squark masses which enhance $\chi q \stackrel{\tilde{q}}{\rightarrow} \chi q$ in $\sigma_{\chi-p}^{\text {spin }}$, then mainly to the decrease of $\mu$ leading to a larger higgsino fraction in the neutralino which enhance $\chi q \stackrel{Z}{\rightarrow} \chi q$ in $\sigma_{\chi-p}^{\text {spin }}$. Moreover the larger higgsino fraction also favours the neutralino annihilations into the $\chi \chi \rightarrow W^{+} W^{-}, Z Z$ and $\chi \chi \rightarrow t \bar{t}$ channels which give harder neutrino spectra than $\chi \chi \rightarrow b \bar{b}$. This is illustrated on figures 5b, 5 d , 5e, 6b, 6 d, 6e and 77b, 77d, 77e. This enhancement is not as peaked in $x$ as for direct detection but remains maximum as long as the higgsino fraction dominates the neutralino composition. However the relic density becomes very small when $x$ is further lowered. For these extreme low values of $M_{3}$ with small relic density, the enhancement of the muon fluxes coming from neutralino annihilation in the centre of the Earth (enhanced by $\sigma_{\chi-p}^{s c a l}$ and $\sigma_{\chi-\chi}^{A}$ ) can become significant but usually remains beyond reach of current and next generation neutrino telescopes' sensitivities.

To summarise, we have shown it is possible, by lowering $\left.M_{3}\right|_{G U T}$, to decrease the neutralino relic density to the desired cosmological value for any CMSS Model even for $m_{0}$ and $m_{1 / 2}$ as large as several TeV. The value of $\left.M_{3}\right|_{G U T}$ necessary to get a relic density $\Omega_{\chi} h^{2} \sim 0.1-0.2$ mainly depends on $m_{1 / 2}: 0.5 m_{1 / 2}<M_{3}<m_{1 / 2}$.

In the CMSSM, the main neutralino annihilation channels can be regrouped in two sets: the pseudo-scalar exchange $\chi \chi \stackrel{A}{\rightarrow} b \bar{b}$ and the processes directly proportional to the neutralino higgsino fraction $\chi \chi \stackrel{Z}{\rightarrow} t \bar{t}, \chi \chi \stackrel{\chi_{i}^{+}}{\rightarrow} W^{+} W^{-}$and $\chi \chi \stackrel{\chi_{i}}{\rightarrow} Z Z$. As we have seen, the decrease of the $\left.M_{3}\right|_{G U T}$ parameter influences many MSSM parameters through the RGE and leads to both a decrease of $m_{A}$ and an increase of the neutralino higgsino fraction by the decrease of $\mu$. So the dominant neutralino annihilation process of any CMSS Model can be enhanced in order to obtain the good value for the relic density.

Varying the $x$ value allows to have $\Omega_{\chi} h^{2} \sim 0.15$ in the whole shown $\left(m_{0}, m_{1 / 2}\right)$ plane. For $\tan \beta<50$, where the $A$ pole is not present in the neutralino annihilation, the interesting values of $x$ range between $\sim 0.8-1$ along the zone with a correct 


$$
A_{0}=0 ; \tan \beta=45 ; \mu>0 ; M_{3} / m_{1 / 2}=0.63
$$

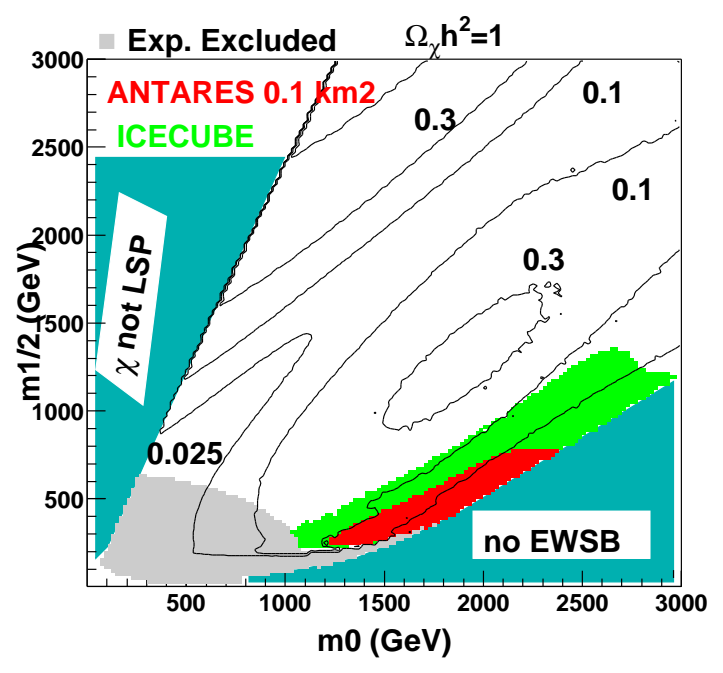

a)

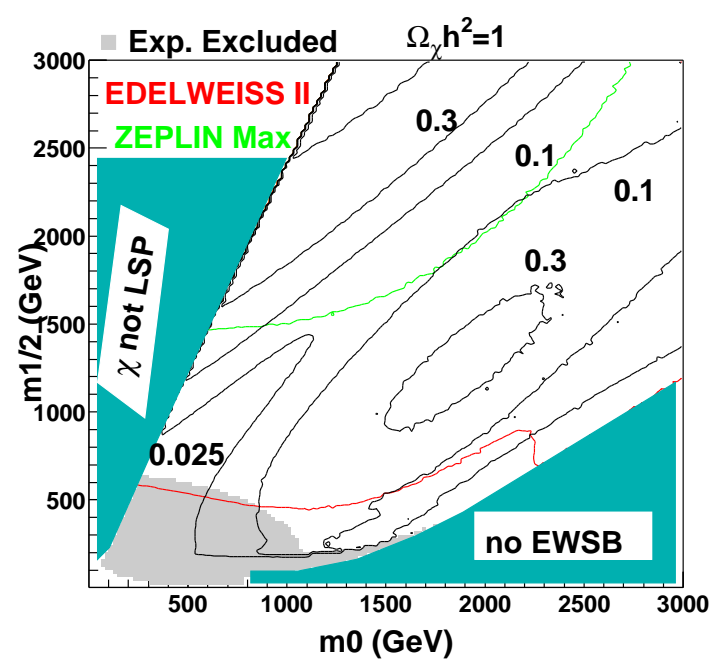

b)

Figure 9: Neutralino detection potential in the $\left(m_{0}, m_{1 / 2}\right)$ plane for $x=M_{3} / m_{1 / 2}=$ 0.63 for a) neutrino telescopes looking at muon fluxes coming from the Sun and b) direct detection experiments.

neutralino relic density in the CMSSM and down to $x \sim 0.5-0.6$ for large $m_{1 / 2}>$ $2000 \mathrm{GeV}$ and $m_{0}<2000 \mathrm{GeV}$. At the centre of the shown $\left(m_{0}, m_{1 / 2}\right)$ plane, a quite generic $x$ value is found

$$
\left.M_{3}\right|_{G U T} \sim 0.6( \pm 0.1) m_{1 / 2}+\operatorname{corrections}\left(m_{0}, \tan \beta, m_{b}\right)
$$

permitting to get close to the $A$ pole or at least to adjust the couple $\left(m_{A}, z_{11(2)} z_{13(4)}\right)$ and thus the $\chi \chi \stackrel{A}{\rightarrow} b \bar{b}$ channel in order to get $\Omega_{\chi} h^{2} \sim 0.1-0.2$. These $x$ values also favour the direct detection yields through the $\chi q \stackrel{H}{\rightarrow} \chi q$ process. Moreover, the decrease of $M_{3}$ increases the neutralino higgsino fraction, so that a typical value $M_{3} / m_{1 / 2} \lesssim 0.8$ enlarges the "focus-point" corridor at large $m_{0}$ as well as the region of the parameter space accessible to direct and indirect detection. Notice that all $x$ values discussed above lie well above $x=0.16$, below which the gluino becomes the LSP.

Two examples with a fixed ratio $M_{3} / m_{1 / 2}$ are shown on figures 9 and 10 where regions with interesting relic density and experiment sensitivity areas are vastly improved with respect to the CMSSM case [4], especially noticing the larger $m_{1 / 2}$ range shown.

\section{Links to SUSY breaking :}

From the above discussions, $\left.M_{3}\right|_{G U T}$ appears as the most relevant parameter in SUSY non-universal models as far as neutralino detection is concerned and we have defined 


$$
A_{0}=0 ; \tan \beta=10 ; \mu>0 ; M_{3} / m_{1 / 2}=0.55
$$

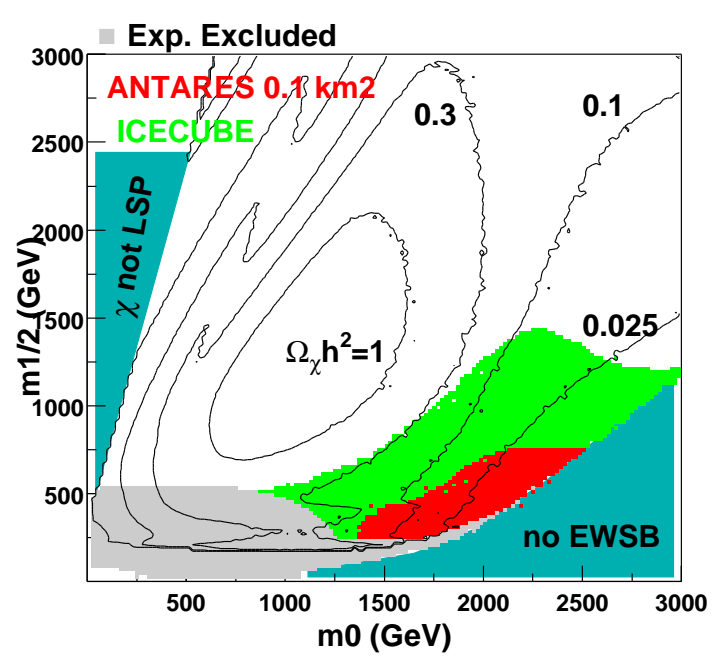

a)

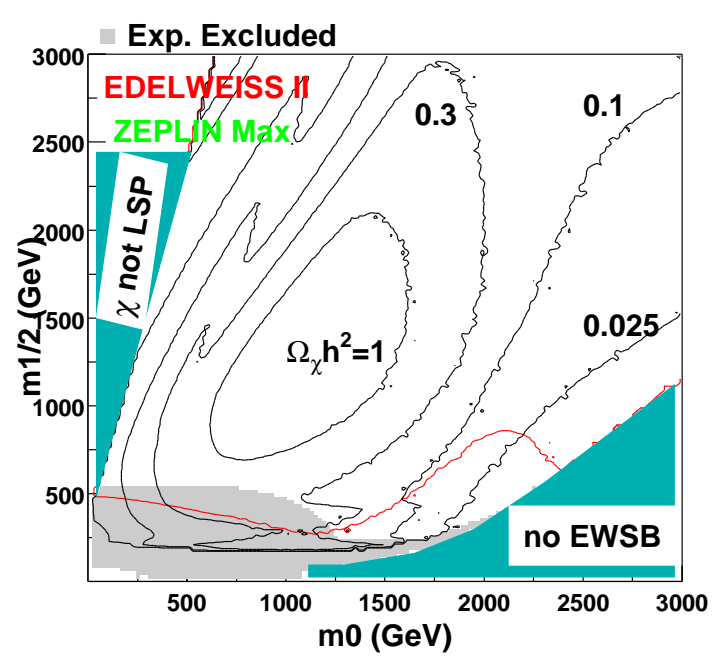

b)

Figure 10: Neutralino detection potential in the $\left(m_{0}, m_{1 / 2}\right)$ plane for $\tan \beta=10$ with $x=M_{3} / m_{1 / 2}=0.55$ for a) neutrino telescopes looking at muon fluxes coming from the Sun and $\mathrm{b}$ ) direct detection experiments (For this value of $x$, Zeplin Max covers the whole shown plane).

its departure from universality by the $x=\left.M_{3}\right|_{G U T} / m_{1 / 2}$ ratio. In line with the logics of minimising the deviations to universality, we can map this single parameter $x$ into a single combination of the $\mathbf{2 4 , 7 5}$ and $\mathbf{2 0 0}$ representations:

$$
\text { non-univ }=\mathrm{CMSSM}+c_{24} 24+c_{75} 75+c_{200} 200
$$

which leads for the relative values of the gaugino masses $\left(M_{1}: M_{2}: M_{3}\right)$ at $M_{G U T}$ to

$$
(1: 1: x)_{\text {non univ }}=(1: 1: 1)_{\mathrm{CMSSM}}+(0: 0: x-1)_{c_{24} \mathbf{2 4}+c_{75} \mathbf{7 5}+c_{200} \mathbf{2 0 0}}
$$

From table 1, we then see that the coefficients must take the values:

$$
c_{24}=\frac{20}{63}(x-1) ; c_{75}=\frac{14}{63}(x-1) ; c_{200}=\frac{9}{63}(x-1) .
$$

giving for the typical value of interest $x=0.6: c_{24} \simeq-0.13 ; c_{75} \simeq-0.09$; $c_{200} \simeq-0.06$, corresponding to quite small coefficients indeed.

A possible origin of such lower $M_{3}$ values might otherwise be found in anomaly mediated SUSY breaking (AMSB) schemes. Even if the usual derivation of AMSB spectra leads to wino LSP's 41, 42], other patterns have been found [43] where the gluino can become lighter, to the point of having a gluino LSP in extreme cases. An exploration of this connection in the general framework of [44 would be interesting. 


\section{Low energy effective parameterisation of the MSSM}

We have explored the grand unification SUSY models favourable to the indirect detection of neutralino dark matter with neutrino telescopes. This kind of study has previously been performed in the low energy MSSM [1, 45]. It is obvious that such a low energy approach offers more free parameters (in particular $\mu$ ) to play with, and it thus becomes much easier to obtain heavy neutralino with a strong higgsino component. The main difference between the grand unification and the low energy approaches comes from the absence of neutralino annihilation into Higgs bosons for the GUT models, due to the RGE evolution of the SUSY parameters and to the mass spectrum hierarchy obtained. This possibility of lighter scalars (Higgs and squarks of first generation) in the low energy approach also sometimes enables fluxes from the Earth to be significant. However these low energy effective models are less consistent and do not take into account some nice features of SUSY GUT models such as the radiative electroweak symmetry breaking or the avoidance of Landau poles and CCB minima.

We propose here to derive a low energy effective parameterisation of SUSY GUT models which appear to be favourable for the neutrino indirect detection of neutralinos. All these models present indeed the same characteristics:

- a non-vanishing neutralino higgsino fraction $f_{H}: 0.1 \lesssim f_{H} \lesssim 0.4$ (see figures 2 and 5 , 6, (7),

- $\chi \chi \stackrel{\chi_{i}^{+}, \chi_{i}}{\rightarrow} W^{+} W^{-}, Z Z$ or $\chi \chi \stackrel{Z}{\rightarrow} t \bar{t}$ dominant neutralino annihilation channels which are very efficient in order to get both a good value of the neutralino relic density and hard neutrino spectra.

One notices that all the physics which governs these processes only involves neutralinos and/or charginos on top of standard particles. Therefore a minimal description assumes all scalars to be heavy and decoupled. The remaining necessary parameters are those defining the neutralino sector : $M_{1}, M_{2}, \mu$ and $\tan \beta$. Moreover the unification relations and the RGE evolutions lead to $M_{2} \simeq 2 M_{1}$, while a neutralino higgsino fraction in the range $0.1 \lesssim f_{H} \lesssim 0.4$ implies (see figure 11)

$$
1.4 \times 10^{-4} M_{1}^{2}+0.83 M_{1}+60 \lesssim \mu \lesssim 1.5 \times 10^{-4} M_{1}^{2}+0.8 M_{1}+150
$$

with negligible dependence in $\tan \beta$ (see twin curves of figure 11).

A SUSY GUT model satisfying at low energy: $M_{\text {scal }} \gg M_{1}, M_{2} \simeq 2 M_{1}$ and $\mu$ in the range indicated by equation (5.1) gets a cosmologically favoured value of the neutralino relic density and large muon fluxes coming from neutralino annihilation in the Sun. For a scan of low energy models with $50<M_{1}<1000 \mathrm{GeV}, M_{\text {scal }} \gg M_{1}$, 


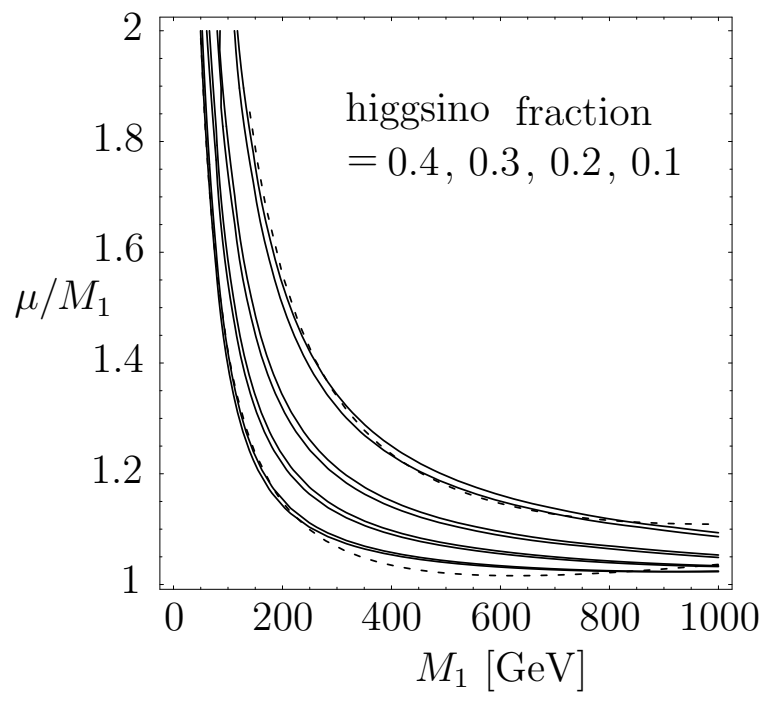

Figure 11: Analytical iso higgsino fractions for $\tan \beta=10$ (lower twin curve) and 50 (upper twin curve) shown as full lines, and the fitted functions described in equation (5.1) for $f_{H}=0.4$ (lower left) and $f_{H}=0.1$ (upper right) shown as dashed lines.

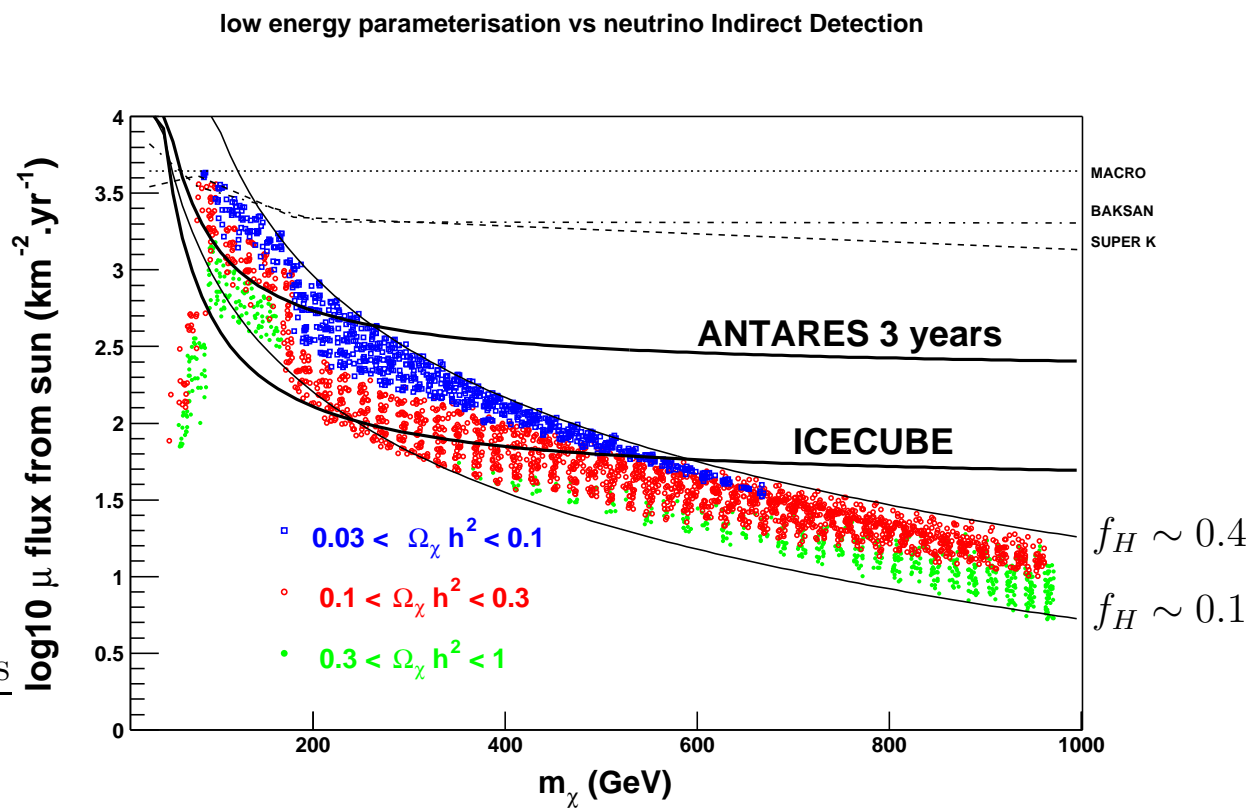

Figure 12: Low energy parameterisation of RGE models versus experiment sensitivities. The thin full lines are the fitted functions of equation (5.2). 
$\tan \beta=5,10,20,30,40,50,60$, and $\mu$ constrained by equation (5.1) to get $0.1 \lesssim f_{H} \lesssim$ 0.4 , we find the following range for the solar $\mu$ flux (see figure 12)

$$
f_{H} \sim 0.1 \leftrightarrow 5 \times 10^{6} \frac{e^{50 / m_{\chi}}}{m_{\chi}^{2}} \lesssim \log _{10}\left[\mu \text { flux }_{\odot}\right] \lesssim 1.5 \times 10^{7} \frac{e^{180 / m_{\chi}}}{m_{\chi}^{2}} \leftrightarrow f_{H} \sim 0.4
$$

We thus see that independently of their high energy scale realisation, the most favourable models are within reach of the next generation $\left(\mathrm{km}^{3}\right)$ of neutrino telescopes through the detection of neutrino fluxes from the Sun, up to masses of the order of $450 \mathrm{GeV}$ for the most interesting relic densities.

\section{Conclusion}

In this paper, we have explored departures from CMSSM universality and singled out those most interesting for neutralino dark matter detection. The scalar sector can be of interest to adjust the neutralino relic density by opening sfermions coannihilation processes, but does not lead to increased detection rates because of the RGE evolution of first generation soft terms. The most determining parameters are the gaugino masses $\left.M_{2}\right|_{G U T}$ and particularly $\left.M_{3}\right|_{G U T}$ which respectively increase the wino and the higgsino content of the neutralino when lowered away from their universal values. The higgsino component is more efficient than the wino one to improve the detection rates, making of $\left.M_{3}\right|_{G U T}$ the most relevant degree of freedom, as its value also affects the whole MSSM spectrum 24]. Such models with lower $\left.M_{3}\right|_{G U T}$ values have a better relic abundance and are much more promising from a detection point of view, with rates increased by several orders of magnitude compared to the universal case. Some naturalness objections could be addressed on the high values of $m_{0}$ and $m_{1 / 2}$ commonly used in the literature. However it should be noticed that lower values of $M_{3}$ tend to be more natural 46. To finish, all the models with large neutrinos fluxes studied here tend to have small SUSY contribution to the $\mu$ anomalous magnetic moment $\left(a_{\mu}^{\mathrm{SUSY}} \simeq 0\right.$ ), which might exclude them, but not before the $a_{\mu}$ value of the Standard Model itself is clearly ruled out.

\section{Acknowledgments}

We thank the "GdR Supersymétrie" of the French CNRS for its support, and in particular acknowledge the early fruitful exchanges with L. Duflot ${ }^{2}$ inside of the working group "Au delà de l'universalité" initiated by J.-F. Grivaz, whose influential role shows up all the way to the title of the present work. More recently, we would also like to thank G. Belanger, S. Rosier-Lees and R. Arnowitt for useful discussions.

\footnotetext{
${ }^{2}$ http://duflot.home.cern.ch/duflot/GDR/GPS_audela/GPS_audela.html
} 


\section{References}

[1] G. Jungman, M. Kamionkowski, and K. Griest, Supersymmetric dark matter, Phys. Rept. 267 (1996) 195-373, http://arXiv.org/abs/hep-ph/9506380.

[2] Antares, http://antares.in2p3.fr, .

[3] Icecube, http://icecube.wisc.edu/, .

[4] V. Bertin, E. Nezri, and J. Orloff, Neutrino indirect detection of neutralino dark matter in the cmssm, http://arXiv.org/abs/hep-ph/0204135.

[5] J. L. Feng, K. T. Matchev, and T. Moroi, Focus points and naturalness in supersymmetry, Phys. Rev. D61 (2000) 075005, http://arXiv.org/abs/hep-ph/9909334.

[6] A. Djouadi, J. Kneur, and G. Moultaka, Suspect program, http://www.lpm.univ-montp2.fr:6714/ kneur/suspect/, .

[7] A. Djouadi, J. Kneur, and G. Moultaka, Suspect: A fortran code for the supersymmetric and higgs particle spectrum in the mssm, hep-ph/0211331.

[8] P. Gondolo, J. Edsjo, L. Bergstrom, P. Ullio, and T. Baltz, Darksusy program, http://www.physto.se/ edsjo/darksusy/, .

[9] MACRO Collaboration, T. Montaruli, Search for wimps using upward-going muons in macro, proceeedings of the 26th icrc in salt lake city, hep-ex/9905021, http://arXiv.org/abs/hep-ex/9905021.

[10] O. V. Suvorova, Status and perspectives of indirect search for dark matter, published in tegernsee 1999, beyond the desert 1999, http://arXiv.org/abs/hep-ph/9911415.

[11] Super-Kamiokande Collaboration, A. Habig, An indirect search for wimps with super-kamiokande, contributed to 27th icrc, hamburg, germany, 7-15 aug 2001., http://arXiv.org/abs/hep-ex/0106024.

[12] L. Thompson, Dark matter prospects with the antares neutrino telescope, talk given at the conference dark 2002, cape town, south africa 4-9 feb, .

[13] J. Edsjo, Swedish astroparticle physics, talk given at the conference 'partikeldagarna', uppsala, sweden, march 6 , .

[14] CDMS Collaboration, R. Abusaidi et. al., Exclusion limits on the wimp nucleon cross-section from the cryogenic dark matter search, Phys.Rev.Lett. 84 (2000) 5699-5703, http://arXiv.org/abs/astro-ph/0002471.

[15] A. Benoit et. al., Improved exclusion limits from the edelweiss wimp search, Phys. Lett. B545 (2002) 43-49, astro-ph/0206271. 
[16] G. Chardin, Edelweiss dark matter search, talk given at the school and workshop on neutrino particle astrophysics, les houches 21 jan -1st feb 2002, .

[17] N. Spooner, New limits and progress from the boulby dark matter programme, talk given at the school and workshop on neutrino particle astrophysics, les houches 21 jan -1rst feb, .

[18] R. McPherson, Lessons and loopholes from susy searches at lep, talk given at susy 02, desy hambourg 17-23 juin, 2002, .

[19] E. Gross, Higgs-boson physics at lep, talk given at susy 02, desy hambourg 17-23 juin, 2002, .

[20] B. Allanach, S. Kraml, and W. Porod, Comparison of susy mass spectrum calculations, http://arXiv.org/abs/hep-ph/0207314.

[21] M. Ciuchini, G. Degrassi, P. Gambino, and G. F. Giudice, Next-to-leading qcd corrections to $b-\dot{z} x / s$ gamma: Standard model and two-higgs doublet model, Nucl. Phys. B527 (1998) 21-43, http://arXiv.org/abs/hep-ph/9710335.

[22] Particle Data Group Collaboration, D. E. Groom et. al., Review of particle physics, Eur. Phys. J. C15 (2000) 1-878.

[23] M. Knecht and A. Nyffeler, Hadronic light-by-light corrections to the muon g-2: The pion-pole contribution, Phys. Rev. D65 (2002) 073034, http://arXiv.org/abs/hep-ph/0111058.

[24] D. Kazakov and G. Moultaka, Analytical study of non-universality of the soft terms in the mssm, Nucl. Phys. B577 (2000) 121-138, http://arXiv.org/abs/hep-ph/9912271.

[25] D. I. Kazakov, Exploring softly broken susy theories via grassmannian taylor expansion, Phys. Lett. B449 (1999) 201-206, http://arXiv.org/abs/hep-ph/9812513.

[26] C. T. Hill, C. N. Leung, and S. Rao, Renormalization group fixed points and the higgs boson spectrum, Nucl. Phys. B262 (1985) 517.

[27] J. R. Ellis, T. Falk, K. A. Olive, and M. Srednicki, Calculations of neutralino stau coannihilation channels and the cosmologically relevant region of mssm parameter space, Astropart. Phys. 13 (2000) 181-213, http://arXiv.org/abs/hep-ph/9905481.

[28] C. Boehm, A. Djouadi, and M. Drees, Light scalar top quarks and supersymmetric dark matter, Phys. Rev. D62 (2000) 035012, http://arXiv.org/abs/hep-ph/9911496.

[29] A. Djouadi, M. Drees, and J. L. Kneur, Constraints on the minimal supergravity model and prospects for susy particle production at future linear $e+e$-colliders, JHEP 08 (2001) 055, http://arXiv.org/abs/hep-ph/0107316. 
[30] J. R. Ellis, K. A. Olive, and Y. Santoso, Calculations of neutralino stop coannihilation in the cmssm, cern-th-2001-339, umn-th-2032-01, tpi-minn-01-50, hep-ph/0112113, http://arXiv.org/abs/hep-ph/0112113.

[31] V. Berezinsky, B. A., E. J. R., F. N., M. G., and S. S., Searching for relic neutralinos using neutrino telescopes, Astropart. Phys. 5 (1996) 333-352, hep-ph/9603342].

[32] J. R. Ellis, T. Falk, K. A. Olive, and Y. Santoso, Exploration of the mssm with non-universal higgs masses, hep-ph/0210205.

[33] V. D. Barger, F. Halzen, D. Hooper, and C. Kao, Indirect search for neutralino dark matter with high energy neutrinos, Phys. Rev. D65 (2002) 075022, http://arXiv.org/abs/hep-ph/0105182.

[34] J. R. Ellis, K. A. Olive, and Y. Santoso, The mssm parameter space with non-universal higgs masses, Phys. Lett. B539 (2002) 107-118, hep-ph/0204192.

[35] J. Amundson et. al., Report of the supersymmetry theory subgroup, http://arXiv.org/abs/hep-ph/9609374.

[36] P. Nath and R. Arnowitt, Non-universal soft susy breaking and dark matter, Phys. Rev. D56 (1997) 2820-2832, hep-ph/9701301.

[37] A. Corsetti and P. Nath, Gaugino mass nonuniversality and dark matter in sugra, strings and d brane models, Phys. Rev. D64 (2001) 125010, hep-ph/0003186.

[38] J. R. Ellis, A. Ferstl, and K. A. Olive, Re-evaluation of the elastic scattering of supersymmetric dark matter, Phys. Lett. B481 (2000) 304-314, http://arXiv.org/abs/hep-ph/0001005.

[39] G. Belanger, F. Boudjema, F. Donato, R. Godbole, and S. Rosier-Lees, Susy higgs at the lhc: Effects of light charginos and neutralinos, Nucl. Phys. B581 (2000) 3-33, http://arXiv.org/abs/hep-ph/0002039.

[40] A. Birkedal-Hansen and B. D. Nelson, The role of wino content in neutralino dark matter, Phys. Rev. D64 (2001) 015008, http://arXiv.org/abs/hep-ph/0102075.

[41] T. Moroi and L. Randall, Wino cold dark matter from anomaly-mediated susy breaking, Nucl. Phys. B570 (2000) 455-472, http://arXiv.org/abs/hep-ph/9906527.

[42] K. Huitu, J. Laamanen, and P. N. Pandita, Sparticle spectrum and constraints in anomaly mediated supersymmetry breaking models, Phys. Rev. D65 (2002) 115003, http://arXiv.org/abs/hep-ph/0203186.

[43] C. H. Chen, M. Drees, and J. F. Gunion, A non-standard string/susy scenario and its phenomenological implications, Phys. Rev. D55 (1997) 330-347, http://arXiv.org/abs/hep-ph/9607421. 
[44] P. Binetruy, M. K. Gaillard, and B. D. Nelson, One loop soft supersymmetry breaking terms in superstring effective theories, Nucl. Phys. B604 (2001) 32-74, http://arXiv.org/abs/hep-ph/0011081.

[45] J. Edsjo, Aspects of neutrino detection of neutralino dark matter, http://arXiv.org/abs/hep-ph/9704384.

[46] G. L. Kane and S. F. King, Naturalness implications of lep results, Phys. Lett. B451 (1999) 113-122, http://arXiv.org/abs/hep-ph/9810374. 